\title{
Proposal of A New Bois Noir Epidemiological Pattern Related to 'Candidatus Phytoplasma Solani' Strains Characterized by A Possible Moderate Virulence in Tuscany
}

\author{
Roberto Pierro ${ }^{1}$, Alessandra Panattoni ${ }^{1}$, Alessandro Passera ${ }^{2}$, Alberto Materazzi ${ }^{1}$, \\ Andrea Luvisi ${ }^{3}{ }^{\mathbb{D}}$, Augusto Loni ${ }^{1}$, Marco Ginanni ${ }^{4}$, Andrea Lucchi ${ }^{1}$, Piero Attilio Bianco ${ }^{2,5, *}$ (D) \\ and Fabio Quaglino 2 (D) \\ 1 Department of Agriculture, Food and Environment (DAFE), University of Pisa, via del Borghetto 80, \\ 56124 Pisa, Italy; rob.pierro@outlook.it (R.P.); alessandra.panattoni@unipi.it (A.P.); \\ alberto.materazzi@unipi.it (A.M.); augusto.loni@unipi.it (A.L.); andrea.lucchi@unipi.it (A.L.) \\ 2 Department of Agricultural and Environmental Sciences, Production, Landscape, Agroenergy (DiSAA), \\ University of Milan, via Celoria 2, 20133 Milano, Italy; alessandro.passera@unimi.it (A.P.); \\ fabio.quaglino@unimi.it (F.Q.) \\ 3 Department of Biological and Environmental Sciences and Technologies, University of Salento, \\ via Provinciale Monteroni, 73100 Lecce, Italy; andrea.luvisi@unisalento.it \\ 4 Agro-Environmental Research Center "Enrico Avanzi" (CiRAA), University of Pisa, via Vecchia di Marina 6, \\ 56122 Pisa Italy; ginanni@tiscali.it \\ 5 Institute for Sustainable Plant Protection, National Research Council (IPSP-CNR), Strada delle Cacce 73, \\ 10135 Turin, Italy \\ * Correspondence: piero.bianco@unimi.it
}

Received: 28 February 2020; Accepted: 6 April 2020; Published: 7 April 2020

check for updates

\begin{abstract}
Bois noir (BN), associated with 'Candidatus Phytoplasma solani' (CaPsol), is the most widespread disease of the grapevine yellows complex worldwide. In this work, BN epidemiology was investigated in a case study vineyard where an unusual CaPsol strain, previously detected only in other host plants, was found to be prevalent in grapevine. Experimental activities included: symptom observation; sampling of symptomatic vines, Auchenorrhyncha specimens, and weeds; molecular detection and typing of CaPsol strains; statistical analyses for determining possible relationships between CaPsol relative concentration, strain type, and symptom severity. Among insects, Reptalus quinquecostatus was the most abundant and was found to be highly infected by CaPsol, while Hyalesthes obsoletus, the main CaPsol vector, was not caught. Moreover, R. quinquecostatus harbored CaPsol strains carrying uniquely the stamp sequence variant $\mathrm{St} 10$, also identified as prevalent in vines and in the majority of weeds, and all the $\sec Y$ variants identified in the vineyard. Statistical analyses revealed that CaPsol strains carrying the St10 variant are not associated with severe symptoms, suggesting their possible moderate virulence. Based on such evidence, a new BN epidemiological pattern related to these CaPsol strains and involving grapevine, R. quinquecostatus, and/or weeds is proposed. Furthermore, the possible presence of other players (vectors and weeds) involved in CaPsol transmission to grapevines was highlighted.
\end{abstract}

Keywords: grapevine yellows; Reptalus quinquecostatus; Vitis vinifera cv. Sangiovese; MLST; stamp; secY

\section{Introduction}

Grapevine yellows (GY) diseases, associated with phytoplasmas, constitute a major threat to viticulture worldwide. Bois noir (BN), one of the most important GY disease, is associated with 
'Candidatus Phytoplasma solani' (CaPsol, taxonomic subgroup 16SrXII-A). CaPsol largely spread in Europe, Mediterranean regions and Iran, and has been sporadically reported from China, Chile, and South Africa [1-3]. In grapevine, BN induces symptoms undistinguishable from those of other GY diseases, such as desiccation of inflorescences, berry shrivel, leaf discolorations, reduction in growth, and irregular ripening of wood [4]. The cixiid Hyalesthes obsoletus Signoret, the main vector of BN [4], acquires CaPsol from its preferred host plants (Convolvulus arvensis L. and Urtica dioica L.), and occasionally transmits it to grapevine, a phytoplasma dead-end host $[5,6]$. Recently, typing of CaPsol strains using different molecular markers improved the knowledge of BN spreading, highlighting the crucial role of Vitex agnus-castus L. and Crepis foetida L. as the main CaPsol source plants for $H$. obsoletus $[7,8]$, and proposing the involvement of other weeds and insects in BN epidemiology $[9,10]$. Moreover, combining field surveys, phytoplasma molecular characterization and transmission trials, Reptalus panzeri (Löw) was reported as a natural vector of CaPsol in Serbian vineyards [11], and another eight alternative vectors of CaPsol to grapevine (Aphrodes makarovi Zachvatkin, Dicranotropis hamata (Boheman), Dictyophara europaea (L.), Euscelis incisus (Kirschbaum), Euscelidius variegatus (Kirschbaum), Laodelphax striatella (Fallen), Philaenus spumarius (L.), and Psammotettix alienus/confinis (Dahlbom)) were identified in northern Italy [12].

Recently, a CaPsol strain, previously detected only in other host plants and characterized by carrying stamp gene sequence variant St10, was found prevalent in $\mathrm{BN}$-affected vineyards in the Chianti Classico area, Tuscany. Due to its widespread distribution and moderate virulence, it was hypothesized that this CaPsol strain coevolved in the Tuscan vineyard agroecosystem, adapting to grapevine and other hosts $[13,14]$. Interestingly, previous studies conducted in vineyards located in the same area revealed the abundant presence of the cixiid Reptalus quinquecostatus (Dufour) [15], found able to transmit CaPsol to feeding medium and to periwinkle and suspected to be a CaPsol vector to grapevine [16,17], and of spontaneous weeds largely infected by CaPsol [18]. Such evidences opened a new intriguing scenario on the epidemiology of BN in Tuscany.

In the present study, conducted in a Sangiovese vineyard located in the Chianti Classico area, a molecular epidemiology approach was applied to investigate such a scenario in order to identify the actors involved in CaPsol diffusion to grapevine, with a particular focus on the strain newly found in association with BN. Moreover, statistical analyses were carried out to find a possible correlation between symptom severity and CaPsol strains.

\section{Results}

Partial results on CaPsol detection and stamp gene based typing in grapevines and Reptalus quinquecostatus (Dufour), included in this study, were preliminarily presented at IPWG Meeting 2019 (Valencia, Spain) [19]. To improve clarity and facilitate the readability and comprehension of the present study, such partial results were included in the manuscript.

\subsection{Sampling and Phytoplasma Detection}

The survey in the vineyard showed an incidence of GY symptoms equal to $6.7 \%$ (48 symptomatic vines out of 715 ). Based on symptom severity, 26 of these vines (54.2\%) exhibiting severe symptoms were inserted in class $3 ; 12$ of these vines (25\%) exhibiting moderate symptoms were inserted in class 2; and 10 of these vines (20\%) exhibiting mild symptoms were inserted in class 1 (Table 1 ). 
Table 1. Symptom severity class, relative abundance, and strain typing of CaPsol strains identified in grapevines located in the Greve in Chianti vineyard.

\begin{tabular}{|c|c|c|c|c|}
\hline \multirow{2}{*}{ Vine Sample ID } & \multirow{2}{*}{$\begin{array}{l}\text { Symptom Severity } \\
\text { Class }^{\text {a }}\end{array}$} & \multicolumn{3}{|c|}{ CaPsol Relative Abundance and Typing ${ }^{b}$} \\
\hline & & $\Delta \mathrm{Cq}^{\mathrm{c}}$ & $\begin{array}{l}\text { stamp Sequence } \\
\text { Variant } \\
\text { d }\end{array}$ & $\begin{array}{l}\text { secY Sequence } \\
\text { Variant }{ }^{\mathrm{d}}\end{array}$ \\
\hline G13 & 1 & 11.3 & St10 & SecY9 \\
\hline G14 & 1 & 10.3 & St10 & - \\
\hline G27 & 1 & 10.4 & St10 & SecY33 \\
\hline G29 & 1 & - & - & - \\
\hline G37 & 1 & 8.36 & St10 & SecY6 \\
\hline G40 & 1 & 9.39 & St10 & - \\
\hline G61 & 1 & - & - & - \\
\hline G70 & 1 & 7.45 & St10 & - \\
\hline G72 & 1 & 7.89 & St10 & - \\
\hline G74 & 1 & 12.4 & St5 & - \\
\hline G4 & 2 & 9.03 & St59 & - \\
\hline G7 & 2 & 5.56 & St10 & - \\
\hline G24 & 2 & 8.35 & St10 & - \\
\hline G34 & 2 & 7.59 & St18 & - \\
\hline G45 & 2 & 10.3 & - & - \\
\hline G55 & 2 & 6.35 & St5 & SecY1 \\
\hline G59 & 2 & 3.66 & St5 & - \\
\hline G60 & 2 & 8.62 & St5 & - \\
\hline G62 & 2 & 3.62 & St10 & SecY6 \\
\hline G68 & 2 & 6.35 & St10 & \\
\hline G71 & 2 & 9.51 & St18 & SecY33 \\
\hline G73 & 2 & 8.99 & St10 & SecY33 \\
\hline G1 & 3 & 3.43 & St10 & - \\
\hline G2 & 3 & 8.17 & St10 & SecY1 \\
\hline G3 & 3 & 8.82 & - & - \\
\hline G6 & 3 & 4.21 & St18 & SecY9 \\
\hline G10 & 3 & 6.86 & St5 & SecY1 \\
\hline G12 & 3 & 12.3 & St5 & SecY1 \\
\hline G11 & 3 & 4.49 & St18 & - \\
\hline G8 & 3 & 9.26 & St5 & - \\
\hline G16 & 3 & 4.81 & St10 & SecY33 \\
\hline G18 & 3 & 6.17 & St18 & - \\
\hline G21 & 3 & 3.65 & St10 & - \\
\hline G22 & 3 & 4.25 & St10 & SecY9 \\
\hline G31 & 3 & 5.44 & St5 & - \\
\hline G32 & 3 & 3.45 & St18 & SecY9 \\
\hline G33 & 3 & 5.91 & St5 & - \\
\hline G38 & 3 & 4.63 & St10 & SecY1 \\
\hline G42 & 3 & 2.14 & St10 & SecY6 \\
\hline G43 & 3 & 3.07 & St10 & - \\
\hline G44 & 3 & 3.87 & St10 & SecY1 \\
\hline G48 & 3 & 7.67 & St18 & - \\
\hline G52 & 3 & 6.76 & St5 & SecY9 \\
\hline G54 & 3 & - & - & - \\
\hline G58 & 3 & 7.25 & St18 & - \\
\hline G67 & 3 & 4.91 & St10 & SecY9 \\
\hline G69 & 3 & 6.08 & St18 & SecY9 \\
\hline G75 & 3 & 8.34 & St5 & - \\
\hline
\end{tabular}

${ }^{\mathrm{a}}$, symptom severity: mild (1); moderate (2); severe (3); ${ }^{\mathrm{b}}$, symbol "-": negative to amplification reaction; ${ }^{\mathrm{c}}, \Delta \mathrm{Cq}$ : normalized value of CaPsol relative quantification; ${ }^{d}$, CaPsol gene sequence variant determined by nucleotide sequence identity value versus sequence variants of the published datasets $[13,14]$. 
CaPsol was detected using real-time PCR in 45 out of 48 symptomatic vines [19] (Table 1). Phytoplasmas belonging to the 16SrI and 16SrV [including Flavescence dorée (FD) phytoplasma] taxonomic groups were not identified in any sample (grapevines, weeds, and insects). Real-time PCR analysis gave no amplification in negative controls (healthy grapevine control plant and reaction mixtures devoid of nucleic acids).

During the field surveys, asymptomatic weeds were collected and identified: leaf samples were collected from 54 weeds of nine species belonging to six families (Table 2). Real-time PCR analysis detected the presence of $\mathrm{CaPsol}$ in 33 out of 54 asymptomatic weeds. In detail, the species showing higher phytoplasma infection rate were Convolvulus arvensis L., Pichris hieracioides L., Sonchus oleraceus L., Clematis vitalba L., and Potentilla reptans L.; Plantago major L. was the sole species found not to be infected. Ammi majus L. and Centaurium erythraea Rafn. were found infected by CaPsol for the first time in the present study (Table 2).

Table 2. CaPsol identification and typing in collected weeds.

\begin{tabular}{|c|c|c|c|c|c|}
\hline Family & Species & $\begin{array}{c}\text { No. of Samples } \\
\text { Infected/Collected }\end{array}$ & Sample ID & $\begin{array}{l}\text { stamp Sequence } \\
\text { Variant }{ }^{a, b}\end{array}$ & $\begin{array}{l}\text { secY Sequence } \\
\text { Variant }^{\mathrm{a}, \mathrm{b}}\end{array}$ \\
\hline Apiaceae & Ammi majus L. & $2 / 5$ & W32 & St10 & - \\
\hline Asteraceae & Centaurium erythraea Rafn. & $1 / 2$ & W23 & St10 & SecY33 \\
\hline Asteraceae & Matricaria chamomilla L. & $1 / 7$ & W31 & St10 & SecY33 \\
\hline \multirow[t]{5}{*}{ Asteraceae } & Pichris hieracioides L. & $7 / 9$ & W39 & St59 & SecY33 \\
\hline & & & W44 & St10 & SecY33 \\
\hline & & & W50 & St10 & - \\
\hline & & & W51 & St10 & SecY1 \\
\hline & & & W65 & St10 & SecY9 \\
\hline \multirow[t]{2}{*}{ Asteraceae } & Sonchus oleraceus L. & $6 / 8$ & W30 & St10 & - \\
\hline & & & W61 & St10 & SecY9 \\
\hline \multirow[t]{10}{*}{ Convolvulaceae } & Convolvulus arvensis $\mathrm{L}$. & $12 / 17$ & W21 & St10 & SecY33 \\
\hline & & & W26 & St10 & SecY33 \\
\hline & & & W28 & St10 & - \\
\hline & & & W37 & St59 & SecY33 \\
\hline & & & W38 & St10 & - \\
\hline & & & W41 & St10 & - \\
\hline & & & W42 & St10 & - \\
\hline & & & W45 & St10 & - \\
\hline & & & W48 & St10 & - \\
\hline & & & W58 & St10 & - \\
\hline
\end{tabular}

${ }^{a}$, symbol " -": negative to amplification reaction; ${ }^{b}, \mathrm{CaPsol}$ gene sequence variant determined by nucleotide sequence identity value versus sequence variants of the published datasets [13,14].

By using sticky traps, 347 Auchenorrhyncha specimens of eight distinct taxonomic groups, four described at genus level and four at species level, were captured [19]. The prevalent taxonomic groups were Reptalus quinquecostatus (Dufour) (Rq) (186 specimens), Phylaenus spumarius (L.) (72 specimens), and Zygina rhamni Ferrari (57 specimens). The other insect taxonomic groups captured were: Psammotettix sp. (21 specimens), Dictyophara europaea (L.) (five specimens), Neophilaenus sp. (four specimens), Cixius sp. (one specimen), and Macrosteles sp. (one specimen). Real-time PCR analysis, conducted on the prevalent species Rq, detected the presence of CaPsol in 76 out of $186 \mathrm{Rq}$ 
specimens [19]. CaPsol-infected samples (45 grapevines, 33 weeds, and $76 \mathrm{Rq}$ ) were utilized in molecular typing analyses.

\section{2. 'Candidatus Phytoplasma Solani' Strain Typing}

Nested PCR products of the genes stamp and secY were amplified from 143 (93\%) and 95 (62\%) out of $154 \mathrm{CaPsol}-$ infected samples, respectively. Regarding the stamp gene, nested PCR products were obtained from 43 out of 45 vines, 67 out of 76 Rq specimens [19], and 33 out of 33 weeds (Tables 1-3). Regarding the sec $Y$ gene, nested PCR products were obtained from 20 out of 45 vines, 15 out of 33 weeds, and 60 out of $76 \mathrm{Rq}$ specimens (Tables 1-3). No amplification was obtained in negative controls.

Table 3. Typing of CaPsol strains identified in R. quinquecostatus specimens captured inside and at the border of the Greve in Chianti vineyard.

\begin{tabular}{ccc}
\hline No. of CaPsol Strains in Rq Specimens & stamp $_{\text {Sequence Variant }}{ }^{\mathbf{b}}$ & sec $_{\text {Y Sequence Variant }}$ S,b $^{\text {, }}$ \\
\hline 12 & St10 & SecY1 \\
4 & St10 & SecY6 \\
16 & St10 & SecY9 \\
28 & St10 & SecY33 \\
7 & St10 & - \\
\hline
\end{tabular}

${ }^{a}$, symbol " -": negative to amplification reaction; ${ }^{\mathrm{b}}, \mathrm{CaPsol}$ gene sequence variant determined by nucleotide sequence identity value versus sequence variants of the published datasets $[13,14]$.

Sequence identity analysis performed on 143 stamp gene amplicons allowed the identification of four sequence variants. Comparison with the stamp gene dataset, previously published in $[13,14]$ and provided in Table S1, revealed that three of these sequence variants were identical to previously reported variants St5, St10, and St18; one variant, identified for the first time in this study and named St 59 , showed a similarity coefficient of $92.6 \%$ ( 35 SNPs) with St10. The nucleotide sequence of St 59 was deposited in the NCBI GenBank repository under the Accession Number MN557212. The distribution of CaPsol stamp sequence variants in grapevines, weeds, and Rq samples was as follows: St10 was prevalent in all examined host plants and the only variant identified in Rq [19]; St5 and St18 were found exclusively in grapevine; lastly, St59 was found in one plant each of grapevine, P. hieracioides, and C. arvensis (Figure 1a).

Sequence identity analysis performed on $95 \mathrm{sec} Y$ gene amplicons allowed the identification of four sequence variants. Comparison with the $\sec Y$ gene dataset, provided in Table S2, revealed that three of these sequence variants were identical to previously reported variants $\operatorname{Sec} Y 1$, SecY6, and SecY9; one variant, identified for the first time in this study and named SecY33, showed a similarity coefficient of $99.7 \%$ with SecY1 and SecY6 (2 SNPs vs. each). The nucleotide sequence of SecY33 was deposited in the NCBI GenBank repository under the Accession Number MN557211. The distribution of CaPsol secY sequence variants in grapevines, weeds, and Rq samples was as follows: SecY1, SecY9, and SecY33 were present in grapevines, weeds, and Rq, while SecY6 was only in grapevines and Rq. In detail, SecY1 and SecY9 were prevalent in grapevines, while SecY33 was prevalent in weeds and Rq (Figure 1b). Concerning the weeds, SecY33 was identified in all the species expect $A$. majus; SecY1 only in P. hieracioides; and SecY9 in A. majus, P. hieracioides, and S. oleraceus (Table 2). 

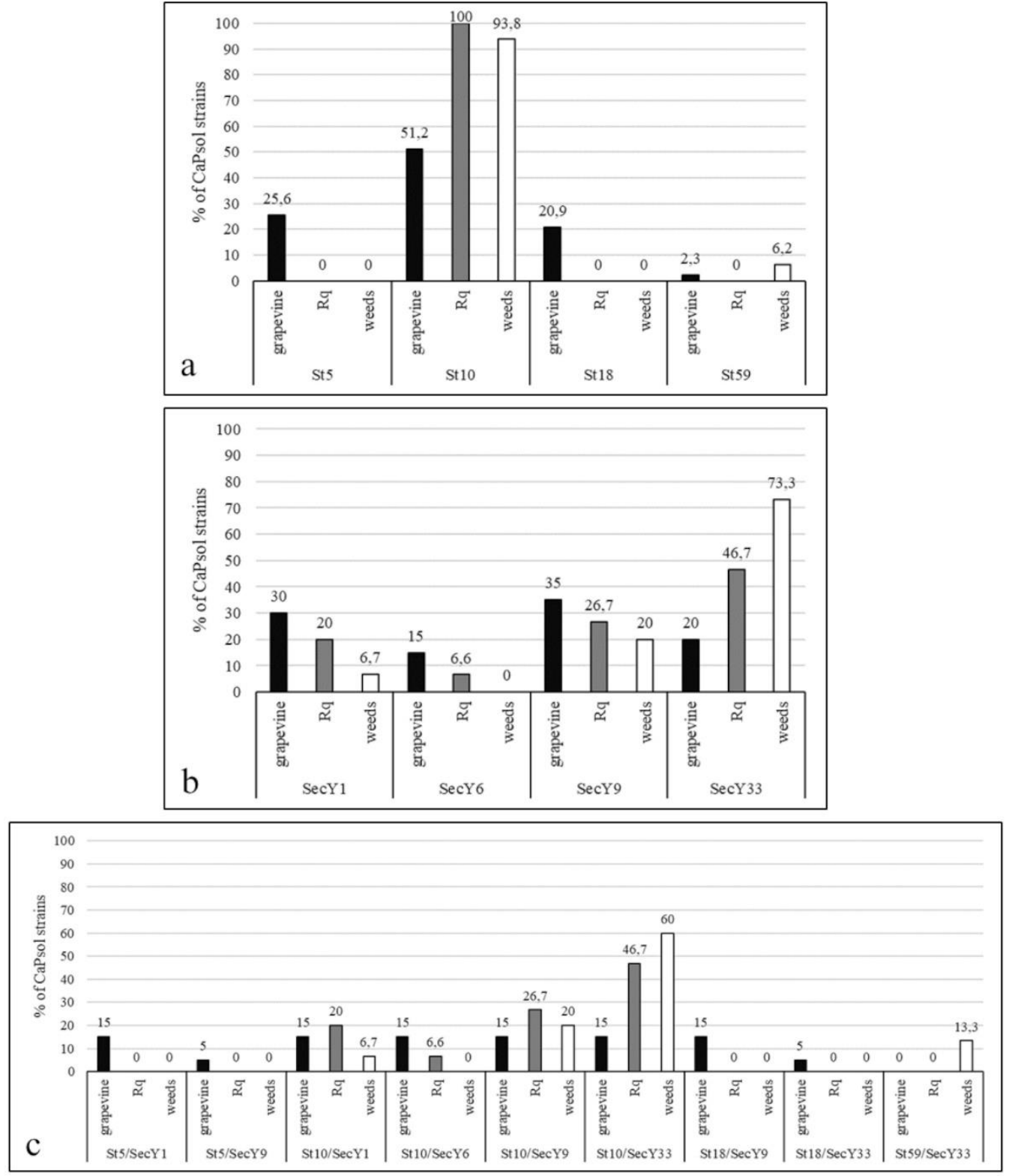

Figure 1. Prevalence (\%) of stamp (a), secY (b) and stamp/secY (c) types of CaPsol strains identified in weeds, R. quinquecostatus specimens and grapevines in the examined vineyard.

The combination between stamp and secY sequence variants (St/SecY type) for each CaPsol strain and the comparison with the provided dataset (Table S3) allowed the identification of nine $\mathrm{St} / \mathrm{SecY}$ types. The distribution of CaPsol St/SecY types in grapevines, weeds, and Rq samples was as follows: St10/SecY1, St10/SecY9, and St10/secY33 were present in grapevines, weeds, and Rq; St10/SecY6 in grapevine and Rq; St5/SecY1, St5/SecY9, St18/SecY9, and St18/SecY33 only in grapevine; and St59/SecY33 only in weeds, specifically P. hieracioides and C. arvensis (Tables 1-3). In particular, St10/SecY33 was the prevalent type in infected weeds and insects, and abundant in grapevine. It was not possible to determine a single prevalent $\mathrm{St} / \mathrm{SecY}$ type in grapevine since six genetically distinct $\mathrm{St} / \mathrm{SecY}$ types shared the same abundance (Figure 1c).

\subsection{Phylogenetic Analyses}

Four main clusters (nettle-related cluster a (divided in two subclusters a1 and a2), and bindweed-related clusters b-I, b-II, and b-III) are identified in the phylogenetic tree generated by the 
analysis of the stamp gene nucleotide sequences of the sequence variants identified in the present study and those reported in the dataset (Table S1). CaPsol strains were identified in the present study and characterized by the stamp sequence variants St5, St10, and the newly reported St59 grouped within the bindweed-related clusters b-I (St10 and St59) and b-II (St5); while the ones carrying the sequence variant St18 were positioned within the nettle-related subcluster a1 (Figure 2).
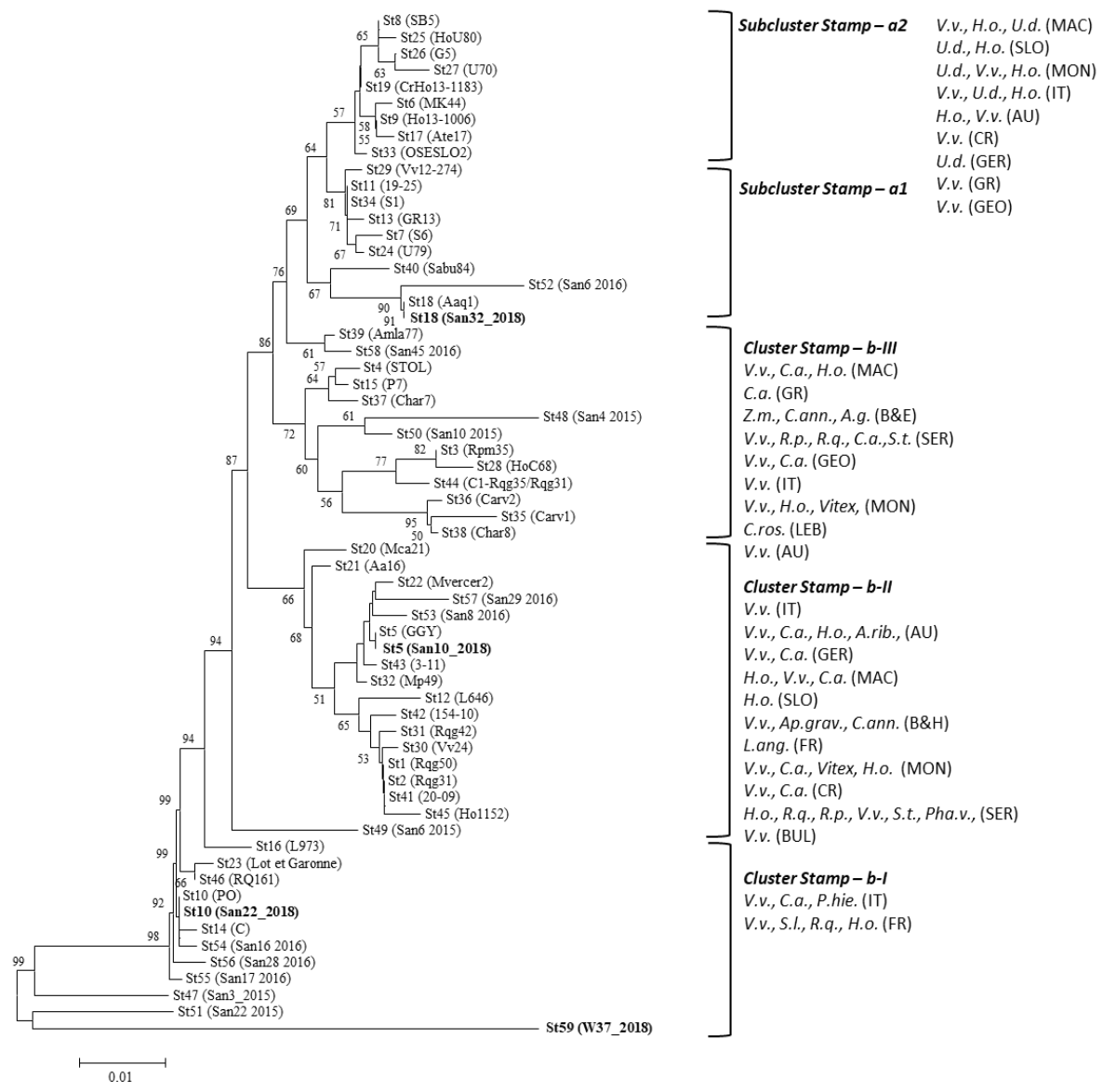

Figure 2. Unrooted phylogenetic tree inferred from stamp gene nucleotide sequences of CaPsol strains representative of stamp sequence variants previously described (Table S1) and identified in this study (Tables 1-3); minimum evolution analysis was performed using the neighbour-joining method and bootstrap replicated 1000 times. Names of strains are reported on the image and nucleotide sequences obtained in this study are in bold. Clusters are shown as delimitated by parentheses. Acronyms within clusters indicated phytoplasma hosts and origin. Hosts: A.g., Apium graveolens; C.ann., Capsicum annum; C.a., Convolvulus arvensis; C.ros., Catharanthus roseus; H.o., Hyalesthes obsoletus; Pha.v., Phaseulus vulgaris; P.hie., Picris hieracioides; R.p., Reptalus panzeri; R.q., Reptalus quinquecostatus; S.l., Solanum lycopersicum; S.t., Solanum tuberosum; U.d., Urtica dioica; Vitex, Vitex agnus-castus; V.v., Vitis vinifera; Z.m., Zea mays. Origin: AU, Austria; B and H, Bosnia and Herzegovina; BUL, Bulgaria; FR, France; GEO, Georgia; GER, Germany; IT, Italy; LEB, Lebanon; MAC, Republic of Macedonia; MON, Montenegro; SER, Serbia; SLO, Slovenia.

Four main clusters, here named $\sec Y-1$ to -4 , have been identified in the phylogenetic tree generated by the analysis of the $\sec Y$ gene nucleotide sequences of the sequence variants identified in the present study and those reported in the dataset (Table S2). CaPsol strains identified in the present study and characterized by the $\sec Y$ sequence variant $\operatorname{Sec} Y 1$ grouped within the cluster $\sec Y-1$; those carrying the sequence variants SecY9 and SecY33 within the cluster sec $Y-2$; those characterized by the sequence variant SecY6 within the cluster secY-3 (Figure 3). 


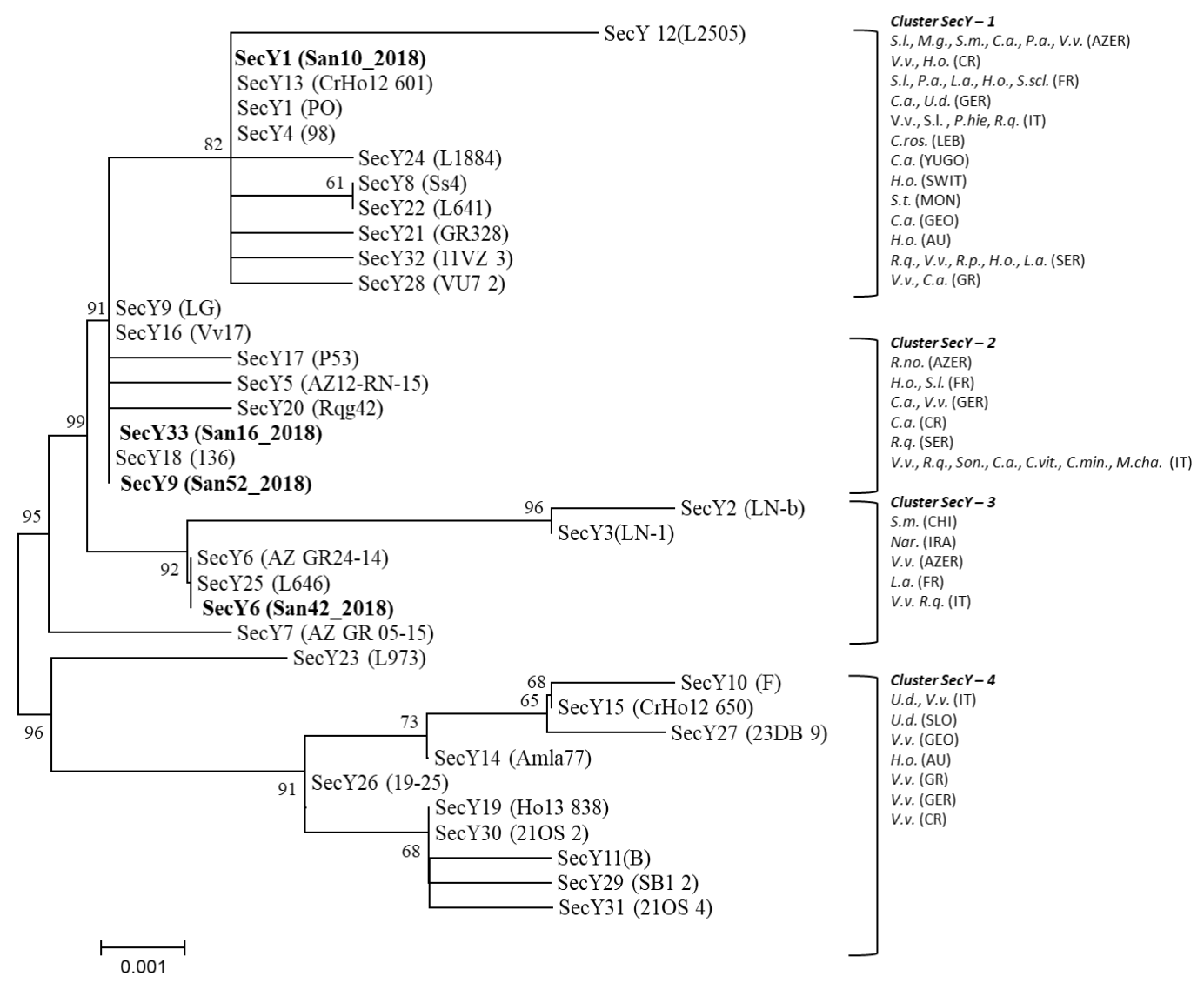

Figure 3. Unrooted phylogenetic tree inferred from sec $Y$ gene nucleotide sequences of CaPsol strains representative of secY sequence variants previously described (Table S2) and identified in this study (Tables 1-3); minimum evolution analysis was performed using the neighbour-joining method and bootstrap replicated 1000 times. Names of strains are reported on the image and nucleotide sequences obtained in this study are in bold. Clusters are shown as delimitated by parentheses. Acronyms within clusters indicated phytoplasma hosts and origin. Hosts: C.a., Convolvulus arvensis; C.min., Centaurea minus; C.ros., Catharanthus roseus; C.v., Clematis vitalba; H.o., Hyalesthes obsoletus; L.a., Lavandula angustifolia; M.cha., Matricaria chamomilla; Nar., Narcissus sp.; Pha.v., Phaseulus vulgaris; P.hie., Picris hieracioides; R.no., Reptalus noahi; R.p., Reptalus panzeri; R.q., Reptalus quinquecostatus; S.l., Solanum lycopersicum; S.m., Salvia miltiorrhiza; Son., Sonchus sp.; S.scl., Salvia sclarea; S.t., Solanum tuberosum; U.d., Urtica dioica; Vitex, Vitex agnus-castus; V.v., Vitis vinifera; Z.m., Zea mays. Origin: AU, Austria; AZER, Azerbaijan; CH, China; CR, Croatia; FR, France; GEO, Georgia; GER, Germany; GR, Greece; IRA, Iran; IT, Italy; LEB, Lebanon; MON, Montenegro; SER, Serbia; SLO, Slovenia; SWIT, Switzerland; YUGO, Yugoslavia.

Four main clusters, here named stamp/sec $Y-1$ to -4 , have been identified in the phylogenetic tree generated by the analysis of the concatenated nucleotide sequences of stamp and $\sec Y$ genes of the $\mathrm{St} / \mathrm{SecY}$ types identified in the present study and those reported in the dataset (Table S3). CaPsol strains identified in the present study and belonging to St/SecY types St5/SecY9, St5/SecY1, St59/SecY33, St10/SecY1, St10/SecY9, St10/SecY6, and St10/SecY33 grouped within the cluster stamp/secY-1; while those belonging to the types St18/SecY33 and St18/SecY9 within the cluster stamp/secY-3 (Figure 4). 


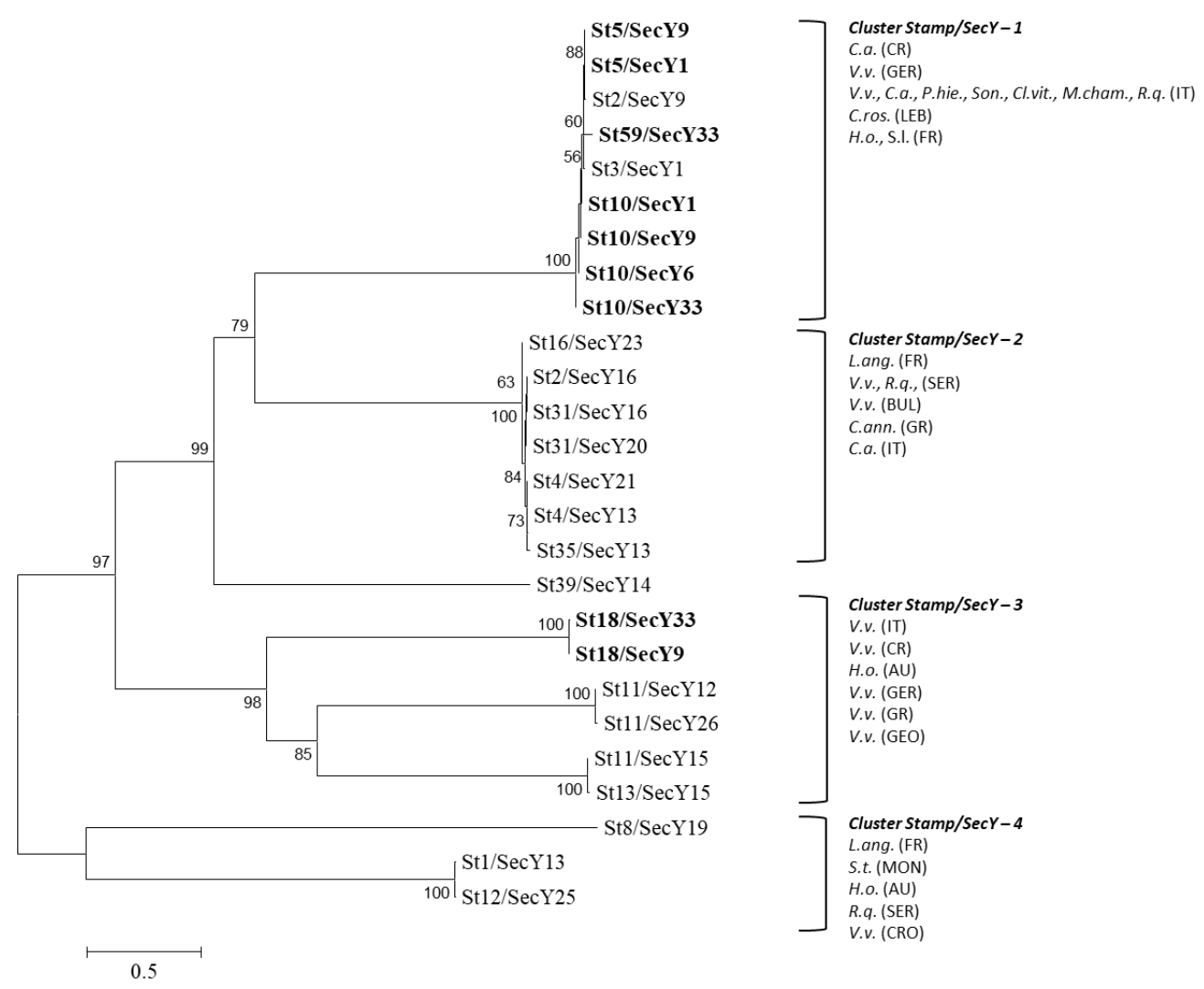

Figure 4. Unrooted phylogenetic tree inferred from stamp/secY gene nucleotide sequences of CaPsol strains representative of stamp/sec $Y$ sequence variants previously described (Table S3) and identified in this study (Tables 1-3); minimum evolution analysis was performed using the neighbor-joining method and bootstrap replicated 1000 times. Names of strains are reported on the image and nucleotide sequences obtained in this study are in bold. Clusters are shown as delimitated by parentheses. Acronyms within clusters indicated phytoplasma hosts and origin. Hosts: C.ann., Capsicum annum; C.a., Convolvulus arvensis; C.ros., Catharanthus roseus; C.v., Clematis vitalba; H.o., Hyalesthes obsoletus; L.a., Lavandula angustifolia; M.cha., Matricaria chamomilla; Nar., Narcissus sp.; Pha.v., Phaseulus vulgaris; P.hie., Picris hieracioides; R.q., Reptalus quinquecostatus; S.l., Solanum lycopersicum; S.m., Salvia miltiorrhiza; Son., Sonchus sp.; S.scl., Salvia sclarea; S.t., Solanum tuberosum; U.d., Urtica dioica; Vitex, Vitex agnus-castus; V.v., Vitis vinifera; Z.m., Zea mays. Origin: AU, Austria; BUL, Bulgaria; CRO, Croatia; FR, France; GEO, Georgia; GER, Germany; GR, Greece; IT, Italy; LEB, Lebanon; MON, Montenegro; SER, Serbia.

\subsection{Relationship between Symptom Severity and 'Candidatus Phytoplasma Solani' Abundance or Strain}

Statistically significant differences between symptom severity observed in symptomatic vines and $\Delta \mathrm{Cq}$ values (relative phytoplasma quantification) were obtained ( $p$ value $=0.000)$. In detail, phytoplasma $\Delta \mathrm{Cq}$ values were: $9.68 \pm 1.72$ in vines showing mild symptoms (class 1 ), $7.32 \pm 2.22$ in vines showing moderate symptoms (class 2), and $5.57 \pm 1.98$ in vines showing severe symptoms (class 3).

On the contrary, no statistically significant differences were observed among $\Delta \mathrm{Cq}$ values obtained from symptomatic grapevines infected by CaPsol strains carrying distinct stamp sequence variants $(p>0.05)$.

No statistically significant differences were obtained in the distribution of CaPsol strains identified in the present study and carrying the stamp sequence variant St10 (common to grapevine, Rq, and weeds) in vines showing mild, moderate, or severe symptoms; while statistically significant differences were obtained in the distribution of $\mathrm{CaPsol}$ strains identified in the present study and carrying the stamp sequence variant St5, St18, and St59 (not common to all examined hosts), found prevalently in 
vines showing severe symptoms (Table 4). The same trend was confirmed by the statistical analysis of the distribution of the overall CaPsol strains, identified in the present $(2017)$ and previous $(2015,2016)$ studies, in vines showing mild, moderate, or severe symptoms (Table 4). Considering the 2017 CaPsol strains carrying the four stamp sequence variants separately, a statistically significant difference was obtained only in the distribution of the strains carrying the nettle-related sequence variant St18, found prevalently in vines showing severe symptoms (Table 4).

Table 4. Distribution of CaPsol strains carrying common and uncommon stamp sequence variants in vines exhibiting mild, moderate, and severe symptoms.

\begin{tabular}{ccccccc}
\hline \multirow{2}{*}{$\begin{array}{c}\text { stamp Sequence } \\
\text { Variant }\end{array}$} & Year & \multicolumn{2}{c}{$\begin{array}{c}\text { No. of CaPsol Strains in Vines with } \\
\text { Different Symptom Severity }\end{array}$} & \multicolumn{2}{c}{ Goodness of Fit } \\
\cline { 2 - 7 } & & Class 1 & Class 2 & Class 3 & Chi Square & P Value \\
\hline common (St10) & 2017 & 7 & 5 & 10 & 2.411 & 0.299 \\
uncommon & $2015-17$ & 12 & 11 & 21 & 3.751 & 0.153 \\
(St5, St18, St59) & 2017 & 1 & 6 & 14 & 12.286 & 0.002 \\
& $2015-17$ & 10 & 21 & 34 & 12.353 & 0.002 \\
\hline
\end{tabular}

\section{Discussion}

Survey on grapevine yellows (GY) symptoms showed that the disease incidence $(6.7 \%)$ in the vineyard in Greve in Chianti was stable in comparison with the previous year (7.2\%), and confirmed the prevalence of diseased vines exhibiting severe (class 3) symptoms ( $54.2 \%$ in 2017 vs. $47.2 \%$ in 2016) (13). Molecular detection of GY phytoplasmas confirmed the absence of flavescence doree (FD) in the examined vineyard. In order to investigate Bois noir (BN) epidemiology and possible differences in the phytoplasma virulence, 'Candidatus Phytoplasma solani' (CaPsol) strains identified in grapevine, insects and weeds were characterized by sequence analyses of variable genes stamp and sec $Y$, largely utilized in previous studies [20-22], and comparison with sequence variant datasets by Pierro and colleagues (Tables S1-S3) [13,14].

As in previous studies on CaPsol multiple gene typing [13,14,21], nested-PCR reactions of stamp gene had the highest amplification rate in samples found to be CaPsol-infected by real-time PCR assays. Molecular typing showed the prevalence of CaPsol strains carrying the stamp gene sequence variants St5, St10, and St18 within the CaPsol population identified in diseased grapevines in 2017, as found in previous years 2015 and 2016 [13]. Thirteen CaPsol strains, each identified in 2015 and 2016 only in one symptomatic vine, were not detected in 2017. These strains, rarely found in grapevines, could have a minor role in BN epidemiology.

The CaPsol strains carrying the stamp gene sequence variant St10, found to be prevalent in BN-affected vines in the examined vineyard in the years 2015, 2016 [13], and 2017 [19], this work was previously detected only in herbaceous host plants. Other studies conducted in vineyards located in the same area revealed the abundant presence of the cixiid Reptalus quinquecostatus (Dufour) (Rq), found able to transmit CaPsol to feeding medium and to periwinkle, and suspected to be a CaPsol vector to grapevine [15-17].

A survey on the Auchenorrhyncha highlighted the large prevalence of $\mathrm{Rq}$ within and at the borders of the studied vineyard, in which no specimens of the main vector Hyalesthes obsoletus Signoret were captured. Moreover, Rq specimens were found to be highly infected (40.8\%) by CaPsol strains characterized uniquely by the stamp sequence variant $S t 10$, as preliminarily reported [19], and by secY sequence variants (SecY1, SecY6, SecY9, and SecY33) identified as also being prevalent in diseased vines and in the majority of the examined weed species. This evidence opens the intriguing hypothesis suggesting the existence of a new epidemiological pattern of $\mathrm{CaPsol}$ in Tuscany vineyards including grapevine, $\mathrm{Rq}$, and weeds. However, transmission trials conducted in controlled conditions demonstrated the capability of Rq to transmit CaPsol to periwinkle but not to grapevine [17]. Due to 
the absence of the experimental proof demonstrating the Rq vectoring activity of CaPsol to grapevine, it was suggested that $\mathrm{Rq}$ could transmit CaPsol only to wild plant reservoirs (mainly bindweeds) present in the vineyard and its surroundings, increasing the inoculum sources for other known vectors (H. obsoletus or Reptalus panzeri (Löw)) able to acquire the phytoplasma from such plants and readily transmit it to grapevine [11,17]. In a previous study carried out in France, Rq was found to carry different $\mathrm{CaPsol}$ strains characterized by distinct stamp sequence variants [17]. Surprisingly, in the examined vineyard $\mathrm{Rq}$ specimens were found to harbor exclusively CaPsol strains carrying the stamp sequence variant $\mathrm{St} 10$. As the protein coded by stamp gene is an antigenic membrane protein, known to be involved in the mechanism determining the phytoplasma-vector specific recognition and interaction [23], it could be hypothesized that Rq population is extremely specialized for interacting with the CaPsol strains carrying the stamp sequence variant St10. Thus, transmission trials are necessary to verify the capability of $\mathrm{Rq}$ to transmit $\mathrm{St} 10 \mathrm{CaPsol}$ strains to grapevine.

Furthermore, the exclusive identification of CaPsol strains carrying the stamp sequence variants St 5 and St18 in grapevine could suggest the presence of other vectors able to transmit to grapevine such $\mathrm{CaPsol}$ strains. It is interesting to note that three insect species, recently identified as alternative vectors of CaPsol to grapevine (Phylaenus spumarius (L.), Psammotettix spp., and Dictyophara europaea (L.)), were found during the survey on Auchenorrhyncha carried out in the vineyard in Greve in Chianti. In detail, transmission trials demonstrated that such insects are able to vector to grapevine CaPsol carrying the sequence variant $\mathrm{St} 5$ [12]. Moreover, based on the preliminary results obtained in this study about the weeds, the absence of St5 and St18 CaPsol strains in the examined weeds can suggest that other insect vectors could be able to acquire and transmit $\mathrm{CaPsol}$ using the infected vines as phytoplasma source.

In this study, the relationship between relative CaPsol concentration ( $\Delta \mathrm{Cq}$ values), CaPsol strain types, and different symptom severity observed in grapevines was investigated. No statistically significant differences were found among $\Delta \mathrm{Cq}$ values of genetically distinct $\mathrm{CaPsol}$ strains. On the other hand, statistical analysis showed that higher relative concentrations of the phytoplasma were associated with vines showing severe symptoms, and lower relative concentrations were associated with vines showing milder symptoms $(p<0.05)$. These data could suggest the existence of a positive relationship between symptom intensity in grapevine and the relative abundance of the phytoplasma. It could be also assumed that higher phytoplasma concentration in plant tissues implies more severe and widespread symptomatology in grapevine, at least for the cv. Sangiovese. Our results are in accordance with previous studies conducted on phytoplasma strains associated with FD [24-26].

Previous studies showed statistically significant differences in the distribution of CaPsol strains, carrying diverse stamp sequence variants, in grapevines with different symptom severity. Thus, it was hypothesized that these CaPsol strains can be characterized by a possible range of virulence $[13,14]$. In the present study, statistical analyses, conducted on CaPsol strains identified in the Greve in Chianti vineyard from 2015 to 2017 [13], this study revealed that CaPsol strains carrying stamp variants St5 and St18, identified in grapevine but not in Rq and in weeds, are associated prevalently with vines exhibiting severe symptoms, suggesting their possible high virulence. On the other hand, no statistically significant differences were found in the distribution of CaPsol strains carrying the stamp variant St10, identified in grapevine, $\mathrm{Rq}$ and weeds, in vines showing mild, moderate, and severe symptoms, suggesting their possible moderate virulence. This evidence reinforces the hypothesis that St10 CaPsol strains, largely prevalent in Tuscany and associated to a possible moderate virulence [13], can be co-evolved with and adapted to the host system grapevine- $\mathrm{Rq}$-weeds in the vineyard agroecosystem in the Chianti Classico area.

In the last few years, numerous studies revealed that the BN epidemiology is more complex than previously postulated, reporting new relevant plant reservoirs (Vitex agnus-castus L. and Crepis foetida L.) for the known CaPsol vectors, and identifying some of the unknown alternative vectors $[7,8,12]$. Here, a new BN epidemiological pattern (grapevine-Rq-weeds) related to St10 CaPsol strains and milder symptoms is proposed. Moreover, the experimental evidence of the present study highlights 
the possible presence of other players (vectors and weeds) involved in CaPsol transmission to and diffusion among grapevines.

\section{Materials and Methods}

\subsection{GY Symptom Observation and Collection of Plant and Insect Samples}

Field surveys were conducted in an organic Sangiovese vineyard (715 plants) located in Greve in Chianti (Chianti Classico area, Florence province), where, in a previous study, the CaPsol strain carrying the stamp gene sequence variant $\mathrm{St10}$ was found to be prevalent in $\mathrm{BN}$-affected grapevines (13). In September 2017, each GY symptomatic vine was visually assessed and attributed to a class of symptom severity, as previously reported [13] About 10 leaves were collected from each symptomatic vine.

In July, weeds reported as potential CaPsol host plants $[9,18]$ and weeds abundant in the vineyard were randomly collected at the inter-row areas and at the borders of the vineyard and identified in accordance with a dichotomous key.

In July, 10 yellow sticky traps were placed inside the vineyard and at its borders based on a regular grid ensuring the homogeneous coverage of the examined area. The traps, positioned on the wire supporting the grapevine canopy (inside the vineyard) and on driven poles at $0.5 \mathrm{~m}$ above the ground (at the borders), were substituted weekly [9] to monitor and capture potential insect vectors. Collected specimens were morphologically identified at genus/species level by stereomicroscope. Insects belonging to the species Reptalus quinquecostatus (Dufour) (Rq), previously found as an abundant putative vector in the examined area [15], were stored in ethanol $95 \%$ at $4{ }^{\circ} \mathrm{C}$.

\subsection{Total nucleic Acids Extraction and Phytoplasma Detection}

Total nucleic acids (TNAs) were extracted from the leaf veins of vine and weed samples with CTAB-based buffer [13]. Insect TNAs were extracted from captured Rq specimens as previously described [27].

Specific detection of phytoplasmas associated with BN, Flavescence dorée (FD, taxonomic group $16 \mathrm{SrV}$ ), and Aster Yellows (AY, taxonomic group 16SrI) was carried out by the amplification of $16 \mathrm{~S}$ ribosomal DNA through TaqMan assay [28], using the Rotor-Gene Q (Qiagen, Germany). TNAs extracted from the leaf veins of a healthy Sangiovese grapevine plant, maintained in the greenhouse of the Department of Agriculture, Food and Environment (University of Pisa, Italy), and a reaction mixture devoid of TNAs were used as negative controls. To avoid contamination in the amplification reaction, no positive controls were utilized. The relative quantification of phytoplasmas in each sample was calculated using the formula: $\Delta \mathrm{Cq}=\mathrm{Cqp}-\mathrm{Cqg}$, where $\Delta \mathrm{Cq}$ is the normalized value, $\mathrm{Cqp}$ is the Cq obtained from amplification of phytoplasmatic $16 S$ rRNA gene, and Cqg is the Cq obtained from amplification of grapevine chaperonin gene, the endogenous control used in the reaction $[28,29]$.

\subsection{Molecular Typing and Phylogeny of 'Candidatus Phytoplasma Solani' Strains}

CaPsol strains detected in vines, weeds, and insects were typed by nucleotide sequence analyses of PCR-based amplicons of the non-ribosomal genomic regions stamp and secY. In detail, fragments of these genes were amplified in nested PCRs [30,31], carried out including the same controls described above for TaqMan assay, and sequenced ( $5 \mathrm{X}$ coverage per base position) by a commercial service (Eurofins Genomics, Germany). Nucleotide sequences of stamp and secY genes were aligned by ClustalW Multiple Alignment and analyzed by Sequence Identity Matrix within the software Bioedit v. 7.0.5.3 [32]. Unique stamp and secY nucleotide sequences, identified in this study, were attributed to sequence variants by their comparison with sequences previously deposited in GenBank and listed in reference datasets previously published $[13,14]$ and provided in the present study (Tables S1-S3). No correspondence was present between the names of sequence variants listed in the reference datasets here utilized and those submitted in the GenBank database in another study [33]. For each stamp and $\sec Y$ sequence variant never reported before this study, one representative nucleotide sequence 
was deposited in the NCBI GenBank. Moreover, the collective type (stamp/secY) was determined by combining the sequence variant of both genes for each sample. A representative CaPsol strain for each stamp, secY, and stamp/secY type was included in phylogenetic analysis generating unrooted phylogenetic trees (Minimum Evolution method, Jukes-Cantor model, Bootstrap replication 1000), using MEGAX software [34].

\subsection{Statistical Analysis}

In a previous work, CaPsol strains, identified in 2015 and 2016 in the same vineyard examined in the present study and carrying distinct stamp gene sequence variants, were found to be differentially distributed in grapevines exhibiting mild, moderate, and severe symptoms. Based on this evidence, it was hypothesized that these CaPsol strains can be characterized by a possible range of virulence [13]. To confirm this hypothesis, the possible association between CaPsol strains carrying distinct stamp gene sequence variants, identified in this (2017) and in the previous study $(2015,2016)$ [13], and symptom severity classes observed in vines were evaluated using Chi square test. To determine if symptom severities were associated with different phytoplasma relative abundance in the vines, $\Delta \mathrm{Cq}$ values were compared between symptom severity classes and CaPsol strain types through one-way ANOVA, followed by Tukey HSD test, using SPSS statistical package for Windows, v. 24.0 (IBM Corporation, Armonk, NY) $(p<0.05)$.

Supplementary Materials: The following are available online at http://www.mdpi.com/2076-0817/9/4/268/s1, Table S1: Sequence variants dataset of the gene stamp among ' $\mathrm{Ca}$. P. solani' strains available in GenBank, Table S2: Sequence variants dataset of the gene secY among 'Ca. P. solani' strains available in GenBank, Table S3: Sequence variants dataset of the stamp/secY-types among 'Ca. P. solani' strains available in GenBank.

Author Contributions: Conceptualization, F.Q., A.M., and P.A.B.; methodology, R.P., A.P. (Alessandra Panattoni), A.L. (Augusto Loni), A.L. (Andrea Luvisi), A.L. (Andrea Lucchi), and M.G.; formal analysis, A.P. (Alessandro Passera); investigation, R.P, A.P. (Alessandra Panattoni), and A.M.; data curation, R.P.; writing - original draft preparation, R.P.; writing - review and editing, F.Q. and A.P. (Alessandro Passera). All authors have read and agreed to the published version of the manuscript.

Funding: This research received no external funding.

Conflicts of Interest: The authors declare no conflict of interest.

\section{References}

1. Bertaccini, A. Grapevine "Bois noir": What is New Under the Sun? In Proceedings of the 5th Bois Noir Workshop, Ljubljana, Slovenia, 18-19 September 2018.

2. Pierro, R.; Semeraro, T.; Luvisi, A.; Garg, H.; Vergine, M.; De Bellis, L.; Gill, H.K. The Distribution of Phytoplasmas in South and East Asia: An Emerging Threat to Grapevine Cultivation. Front. Plant Sci. 2019, 10, 1108. [CrossRef] [PubMed]

3. Quaglino, F.; Zhao, Y.; Casati, P.; Bulgari, D.; Bianco, P.A.; Wei, W.; Davis, R.E. ‘Candidatus Phytoplasma solani', a novel taxon associated with stolbur and Bois Noir related diseases of plants. Intern. J. Syst. Evol. Microbiol. 2013, 63, 2879-2894. [CrossRef] [PubMed]

4. Maixner, M. Transmission of German grapevine yellows (Vergilbungskrankheit) by the planthopper Hyalesthes obsoletus (Auchenorrhyncha: Cixiidae). Vitis 1994, 33, 103-104.

5. Belli, G.; Bianco, P.A.; Conti, M. Grapevine yellows: Past, present and future. J. Plant Path. 2010, 92, 303-326.

6. Langer, M.; Maixner, M. Molecular characterisation of grapevine yellows associated phytoplasmas of the stolbur-group based on RFLP analysis of non-ribosomal DNA. Vitis 2004, 43, 191-200.

7. Kosovac, A.; Radonjić, S.; Hrnčić, S.; Krstić, O.; Toševski, I.; Jović, J. Molecular tracing of the transmission routes of bois noir in Mediterranean Vineyards of Montenegro and experimental evidence for the epidemiological role of Vitex agnus-castus (Lamiaceae) and associated Hyalesthes obsoletus (Cixiidae). Plant Path. 2016, 65, 285-298. [CrossRef] 
8. Kosovac, A.; Jakovljević, M.; Krstić, O.; Cvrković, T.; Mitrović, M.; Toševski, I.; Jović, J. Role of plant-specialized Hyalesthes obsoletus associated with Convolvulus arvensis and Crepis foetida in the transmission of 'Candidatus Phytoplasma solani'-inflicted bois noir disease of grapevine in Serbia. Eur. J. Plant Path. 2019, 153, $183-195$. [CrossRef]

9. Mori, N.; Quaglino, F.; Tessari, F.; Pozzebon, A.; Bulgari, D.; Casati, P.; Bianco, P.A. Investigation on 'bois noir' epidemiology in north-eastern Italian vineyards through a multidisciplinary approach. Ann. Appl. Biol. 2015, 166, 75-89. [CrossRef]

10. Jakovljević, M.; Jović, J.; Krstić, O.; Mitrović, M.; Marinković, S.; Toševski, I.; Cvrkocic, T. Diversity of phytoplasmas identified in the polyphagous leafhopper Euscelis incisus (Cicadellidae, Deltocephalinae) in Serbia: Pathogen inventory, epidemiological significance and vectoring potential. Eur. J. Plant Pathol. 2020, 156, 201-221. [CrossRef]

11. Cvrković, T.; Jović, J.; Mitrović, M.; Krstić, Q.; Toševski, I. Experimental and molecular evidence of Reptalus panzeri as a natural vector of bois noir. Plant Pathol. 2014, 63, 42-53. [CrossRef]

12. Quaglino, F.; Sanna, F.; Moussa, A.; Faccincani, M.; Passera, A.; Casati, P.; Bianco, P.A.; Mori, N. Identification and ecology of alternative insect vectors of 'Candidatus Phytoplasma solani' to grapevine. Sci. Rep. 2019, 9, 19522. [CrossRef] [PubMed]

13. Pierro, R.; Passera, A.; Panattoni, A.; Casati, P.; Luvisi, A.; Rizzo, D.; Bianco, P.A.; Quaglino, F.; Materazzi, A. Molecular typing of 'Bois Noir' phytoplasma strains in the Chianti Classico area (Tuscany, Central Italy) and their association with symptom severity in Vitis vinifera L. cv. Sangiovese. Phytopathol 2018, 108, 362-373. [CrossRef]

14. Pierro, R.; Passera, A.; Panattoni, A.; Rizzo, D.; Stefani, L.; Bartolini, L.; Casati, P.; Luvisi, A.; Quaglino, F.; Materazzi, A. Prevalence of a 'Candidatus Phytoplasma solani' strain, so far associated only with other hosts, in Bois Noir-affected grapevines within Tuscan vineyards. Ann. Appl. Biol. 2018, 173, 202-212. [CrossRef]

15. Trivellone, V.; Pinzauti, F.; Bagnoli, B. Reptalus quinquecostatus (Dufour) (Auchenorrhyncha; Cixiidae) as a possible vector of stolbur-phytoplasma in a vineyard in Tuscany. Redia 2005, 88, 103-108.

16. Pinzauti, F.; Trivellone, V.; Bagnoli, B. Ability of Reptalus quinquecostatus (Hemiptera: Cixiidae) to inoculate stolbur phytoplasma to artificial feeding medium. Ann. Appl. Biol. 2008, 153, 299-305. [CrossRef]

17. Chuche, J.; Danet, J.L.; Salar, P.; Foissac, X.; Thiery, D. Transmission of 'Candidatus Phytoplasma solani' by Reptalus quinquecostatus (Hemiptera: Cixiidae). Ann. Appl. Biol. 2016, 169, 214-223. [CrossRef]

18. Marchi, G.; Cinelli, T.; Rizzo, D.; Stefani, L.; Goti, E.; Della Bartola, M.; Luvisi, A.; Panattoni, A.; Materazzi, A. Occurrence of different phytoplasma infections in wild herbaceous dicots growing in vineyards affected by Bois Noir in Tuscany (Italy). Phytopathol. Mediterr. 2015, 54, 504-515.

19. Pierro, R.; Materazzi, A.; Luvisi, A.; Quaglino, F.; Loni, A.; Lucchi, A.; Panattoni, A. New insights on “bois noir" epidemiology in the Chianti Classico area, Tuscany. Phytopath. Moll. 2019, 9, 39-40. [CrossRef]

20. Murolo, S.; Romanazzi, G. In-vineyard population structure of 'Candidatus Phytoplasma solani' using multilocus sequence typing analysis. Infect. Gen. Evol. 2015, 31, 221-230. [CrossRef]

21. Quaglino, F.; Maghradze, D.; Casati, P.; Chkhaidze, N.; Lobjanidze, M.; Ravasio, A.; Passera, A.; Venturini, G.; Failla, O.; Bianco, P.A. Identification and characterization of new 'Candidatus Phytoplasma solani' strains associated with bois noir disease in Vitis vinifera L. cultivars showing a range of symptoms severity in Georgia, the Caucasus region. Plant Dis. 2016, 100, 904-915.

22. Trivellone, V.; Filippin, L.; Narduzzi-Wicth, B.; Angelini, E. A regional-scale survey to define the known and potential vectors of grapevine yellow phytoplasmas in vineyards South of Swiss Alps. Eur. J. Plant Pathol. 2016, 145, 915-927. [CrossRef]

23. Suzuki, S.; Oshima, K.; Kakizawa, S.; Arashida, R.; Jung, H.Y.; Yamaji, Y.; Nishigawa, H.; Ugaki, M.; Namba, S. Interaction between the membrane protein of a pathogen and insect microfilament complex determines insect-vector specificity. Proc. Natl. Acad. Sci. USA 2006, 103, 4252-4257. [CrossRef] [PubMed]

24. Prezelj, N.; Nikolić, P.; Gruden, K.; Ravnikar, M.; Dermastia, M. Spatiotemporal distribution of flavescence dorée phytoplasma in grapevine. Plant Pathol. 2013, 62, 760-766. [CrossRef]

25. Bulgari, D.; Casati, P.; Quaglino, F.; Bianco, P.A. Endophytic bacterial community of grapevine leaves influenced by sampling date and phytoplasma infection process. BMC Microbiol. 2014, 14, 198. [CrossRef]

26. Roggia, C.; Caciagli, P.; Galetto, L.; Pacifico, D.; Veratti, F.; Bosco, D.; Marzachì, C. Flavescence Dorèe phytoplasma titre in field-infected Barbera and Nebbiolo grapevines. Plant Pathol. 2014, 63, 31-41. [CrossRef] 
27. Marzachì, C.; Veratti, F.; Bosco, D. Direct PCR detection of phytoplasmas in experimentally infected insects. Ann. Appl. Biol. 1998, 133, 4554. [CrossRef]

28. Angelini, E.; Bianchi, G.L.; Filippin, L.; Morassutti, C.; Borgo, M. A new TaqMan method for the identification of phytoplasmas associated with grapevine yellows by real-time PCR assay. J. Microbiol. Methods 2007, 68, 613-622. [CrossRef]

29. Minguzzi, S.; Terlizzi, F.; Lanzoni, C.; Poggi Pollini, C.; Ratti, C. A rapid protocol of crude RNA/DNA extraction for RT-qPCR detection and quantification of 'Candidatus Phytoplasma prunorum'. PLoS ONE 2016, 11, e0146515. [CrossRef]

30. Fabre, A.; Danet, J.L.; Foissac, X. The stolbur phytoplasma antigenic membrane protein gene stamp is submitted to diversifying positive selection. Gene 2011, 472, 37-41. [CrossRef]

31. Fialová, R.; Válová, P.; Balakishiyeva, G.; Danet, J.L.; Sâfárová, D.; Foissac, X.; Navrátil, M. Genetic variability of Stolbur phytoplasma in annual crop and wild plant species in South Moravia. J. Plant Pathol. 2009, 91, 411-416.

32. Hall, T.A. BioEdit: A user-friendly biological sequence alignment editor and analysis program for Windows 95/98/NT. Nucleic Acids Symp. Ser. 1999, 41, 95-98.

33. Sémétey, O.; Gaudin, J.; Danet, J.-L.; Salar, P.; Theil, S.; Fontaine, M.; Krausz, M.; Chaisse, E.; Eveillard, S.; Verdin, E.; et al. Lavender decline in France is associated with chronic infection by lavender-specific strains of "Candidatus Phytoplasma solani". Appl. Environ. Microbiol. 2018, 84, e01507-18. [CrossRef] [PubMed]

34. Kumar, S.; Stecher, G.; Li, M.; Knyaz, C.; Tamura, K. MEGA X: Molecular Evolutionary Genetics Analysis across computing platforms. Mol. Biol. Evol. 2018, 35, 1547-1549. [CrossRef] [PubMed]

(C) 2020 by the authors. Licensee MDPI, Basel, Switzerland. This article is an open access article distributed under the terms and conditions of the Creative Commons Attribution (CC BY) license (http://creativecommons.org/licenses/by/4.0/). 